\title{
RENORMALIZATION IN QED AND QFT WITH A LORENTZ- AND CPT-VIOLATING BACKGROUND
}

\author{
ILYA L. SHAPIRO \\ Departamento de Física, ICE, Universidade Federal de Juiz de Fora \\ Juiz de Fora, Minas Gerais, 36036-900, Brazil \\ E-mail: shapiro@fisica.ufj.br
}

\begin{abstract}
The general features of renormalization and the renormalization group in QED and in general quantum field theories in curved spacetime with additional Lorentz- and CPT-violating background fields are reviewed.
\end{abstract}

\section{Introduction}

There is a growing interest in exploring theories with weakly broken Lorentz and CPT symmetries. The importance of such studies should be clear, especially for those who believe that these important symmetries are exact. Indeed, the unique way to confirm this belief is to assume that they can be actually broken and verify the physical consequences (see Ref. 1 for the latest results in this respect). The general parametrization of all possible ways to break Lorentz and CPT symmetries has been formulated by A. Kostelecký et al. in a series of papers (see, e.g., Ref. 2) and the focus of attention is now naturally shifting to experimental work with different manifestations of the theories with broken symmetries.

An important aspect of theories with broken symmetries is related to their renormalization structure. The reason is that consistency at the quantum level can impose restrictions on the classical theory and, eventually, restrict the space of parameters for experimental verification. On the other hand, such a study can be helpful in establishing the relation between different parameters related to the breaking of Lorentz and CPT symmetries. In what follows we discuss, in particular, the relation between violations of symmetries in the matter and gravitational sectors of the theory. 


\section{Renormalization}

In the well-studied cases of external fields, such as electromagnetic, metric (see, e.g., Ref. 3,4 for the review and further references) and torsion ${ }^{5}$ one can establish the form of possible counterterms by means of covariance and other symmetries from one side and power counting on another side. Naively one may think that these arguments will not work, e.g., for QED with broken Lorentz and CPT symmetries, ${ }^{2}$

$$
\begin{aligned}
S=\int d^{4} x \sqrt{-g} & \left\{\frac{i}{2} \bar{\psi} \Gamma^{\mu} D_{\mu} \psi-\frac{i}{2} D_{\mu}^{\star} \bar{\psi} \Gamma^{\mu} \psi-\bar{\psi} M \psi-\frac{1}{4} F_{\mu \nu} F^{\mu \nu}\right. \\
& \left.-\frac{1}{4}\left(k_{F}\right)_{\mu \nu \alpha \beta} F^{\mu \nu} F^{\alpha \beta}+\frac{1}{2}\left(k_{A F}\right)^{\rho} \varepsilon_{\rho \lambda \mu \nu} A^{\lambda} F^{\mu \nu}\right\} .
\end{aligned}
$$

In the last formula $\Gamma^{\nu}=\gamma^{\nu}+\Gamma_{1}^{\nu}$ and $M=m+M_{1}$, where

$$
\begin{gathered}
\Gamma_{1}^{\nu}=c^{\mu \nu} \gamma_{\mu}+d^{\mu \nu} \gamma_{5} \gamma_{\mu}+e^{\nu}+i f^{\nu} \gamma_{5}+\frac{1}{2} g^{\lambda \mu \nu} \sigma_{\lambda \mu}, \\
M_{1}=a_{\mu} \gamma^{\mu}+b_{\mu} \gamma_{5} \gamma^{\mu}+i m_{5} \gamma_{5}+\frac{1}{2} H_{\mu \nu} \sigma^{\mu \nu} .
\end{gathered}
$$

All these parameters are experimentally proved to be very weak. ${ }^{1}$

The action (1) is perfectly Lorentz and CPT invariant if we treat all new external parameters as fields which transform according to their Lorentz representations. The symmetries get broken if we fix these fields, for instance, considering them to be constant in a given reference frame. However, the renormalization of the theory can be performed in one given frame, and therefore one can safely treat all the fields $a_{\mu}, b_{\mu}, m_{5}, H_{\mu \nu}, c^{\mu \nu}, d^{\mu \nu}, e^{\nu}, f^{\nu}$, and $g^{\lambda \mu \nu}$ as being covariant. For the sake of generality we have included in the action (1) an external metric and, in this way, may use the conventional approach based on covariance.

Consider the renormalization of the theory (1). It is easy to note that the set of external fields includes dimensionless ones in Eq. (2) and dimensional ones in Eq. (3). Furthermore, the renormalization of terms with dimensionless and dimensional terms are problems of different level of complexity.

The form of counterterms constructed from the dimensional fields (3) are strongly restricted by the superficial degree of divergence arguments. At the same time there are no such arguments for the dimensionless fields (2). Without symmetry arguments it is not really possible to take care of renormalization with these terms. Then, the theory with dimensionless fields (2) requires an infinite number of counterterms, hence an infinite number of extra terms in the classical action. However, the situation changes dramatically if we remember that the Lorentz- and CPT-violating terms 
should be very weak. This means we can safely restrict consideration to the terms linear in Lorentz- and CPT-violating fields and all necessary structures can be controlled by symmetry.

According to the arguments presented above, the action of renormalizable theory in curved space includes: (i) covariant generalization of the standard expression, e.g., as given in Eq. (1) for QED and possible nonminimal terms; (ii) vacuum terms depending on the metric and Lorentz- and CPT-violating parameters, but not on matter fields.

The one-loop calculations have been performed for $\mathrm{QED},{ }^{6}$ including in curved space ${ }^{7}$ and they are in a perfect agreement with the consideration presented above. For example, this can be observed in the (incomplete) expression for the vacuum divergences of the 1-loop effective action,

$$
\begin{aligned}
\Gamma_{\mathrm{div}}^{(1)}= & -\frac{\mu^{\epsilon}}{(4 \pi)^{2} \epsilon} \int d^{n} x \sqrt{-g}\left\{R_{\mu \alpha} \nabla_{\rho} \nabla_{\tau} k_{F}^{\tau \alpha \rho \mu}-\frac{1}{6} R \nabla_{\mu} \nabla_{\nu} k_{F}^{\mu \nu}\right. \\
& \left.+\frac{1}{3} R_{\mu \rho \alpha \beta} \nabla^{\beta} \nabla_{\tau} k_{F}^{\tau \mu \alpha \rho}-\frac{1}{12} k_{F}^{\rho \lambda \mu \tau} R R_{\rho \lambda \mu \tau}+\frac{1}{2} k_{F}^{\alpha \rho \tau \lambda} R_{\rho \tau \lambda}^{\mu} R_{\mu \alpha}\right\},
\end{aligned}
$$

where we write $\left(k_{F}\right)^{\mu \lambda \nu}{ }_{\lambda}=\left(k_{F}\right)^{\mu \nu}$.

The expression (4) tells us something essential about renormalization of the theories under discussion. In the curvature-dependent low-energy vacuum sector of the theory one could expect, for dimensional reasons, terms of the form ${ }^{8}$

$$
S_{\text {full }}=\int d^{4} x \sqrt{-g}\left\{\phi R+\phi^{\mu \nu} R_{\mu \nu}+\phi^{\mu \nu \alpha \beta} R_{\mu \nu \alpha \beta}\right\}+S_{\mathrm{HD}}
$$

where the term $S_{\mathrm{HD}}$ represents possible higher derivative terms. As we can see from Eq. (4), the terms presented in Eq. (5) are indeed necessary if we consider a spacetime dependent parameter $k_{F}^{\alpha \beta \mu \nu}$. The introduction of the terms (5) is necessary already at the classical level, for otherwise quantum corrections produce such terms with infinite coefficients which cannot be removed by renormalization.

\section{Renormalization group}

An additional illustration of the relations between different Lorentz- and CPT-violating parameters in the matter and gravity sectors can be obtained within the renormalization group method. The renormalization group equations of the new low-energy sector parameters in the gravitational sector 
have the form ${ }^{7}$

$$
\begin{aligned}
\mu \frac{d \phi}{d \mu} & =\frac{1}{96 \pi^{2}} \nabla_{\mu} \nabla_{\nu} k_{F}^{\mu \nu}, \\
\mu \frac{d \phi^{\mu \nu}}{d \mu} & =-\frac{1}{16 \pi^{2}} \nabla_{\alpha} \nabla_{\beta} k_{F}^{\alpha \mu \beta \nu}, \\
\mu \frac{d \phi^{\mu \nu \alpha \beta}}{d \mu} & =-\frac{1}{48 \pi^{2}} \nabla^{\beta} \nabla_{\lambda} k_{F}^{\lambda \mu \alpha \nu} .
\end{aligned}
$$

These equations show that the order of magnitude of the parameters $\phi$ cannot be too much smaller than that of the variation of the parameter $k_{F}^{\alpha \mu \beta \nu}$. It may be interesting to perform a systematic analysis of these kinds of relations. In fact, for some particular cases the restrictions coming from renormalization group equations can compete with the direct experimental bounds.

\section{Conclusions}

We presented a brief and mainly qualitative review of the renormalization properties of quantum field theories in curved spacetime in the presence of Lorentz- and CPT-violating terms. The study of quantum corrections is useful for formulating the theory in a consistent way.

\section{Acknowledgments}

This work was partially supported by CNPq, FAPEMIG and ICTP.

\section{References}

1. Data Tables for Lorentz and CPT Violation, V.A. Kostelecký and N. Russell, 2013 edition, arXiv:0801.0287v6.

2. D. Colladay, and V.A. Kostelecký, Phys. Rev. D 55, 6760 (1997); Phys. Rev. D 58, 116002 (1998).

3. I.L. Buchbinder, S.D. Odintsov and I.L. Shapiro, Effective Action in Quantum Gravity, IOP Publishing, Bristol, 1992.

4. I.L. Shapiro, Class. Quantum Grav. 25, 103001 (2008).

5. I.L. Shapiro, Phys. Rep. 357, 113 (2002).

6. V.A. Kostelecký, C.D. Lane and A.G.M. Pickering, Phys. Rev. D 65, 056006 (2002); V.C. Zhukovsky, A.E. Lobanov, and E.M. Murchikova, Phys. Rev. D 73, 065016 (2006).

7. G. de Berredo-Peixoto and I.L. Shapiro, Phys. Lett. B642, 153 (2006).

8. V.A. Kostelecký, Phys. Rev. D 69, 105009 (2004). 